\title{
SOME REMARKS ON ABSOLUTE CONTINUITY ON GROUPS
}

\section{GLICKSBERG ${ }^{1}$}

Abstract. The Tam-Larsen result that Radon measures with separable orbits are absolutely continuous is extended somewhat. A consequence is a characterization among finite measures of the absolutely continuous ones as those whose orbits are equi-regular.

Let $G$ be a locally compact group. Some time ago Tam [5], improving on a result of Larsen [4], showed that any Radon ${ }^{2}$ measure $\mu$ on $G$ with a separable orbit under translation is necessarily absolutely continuous. Tam's argument was a natural application of Baire category, and our purpose here is to point out another natural but more measure-theoretic argument which yields a stronger result:

(1) If $\left\{x_{n}\right\}$ is a sequence in $G$ for which $\left\{|\mu|\left(x_{n} E\right)\right\}$ is dense in $\{|\mu|(x E): x \in G\}$ for each compact $|\mu|-$-null $E \subset G$, then $\mu$ is absolutely continuous.

A consequence of the argument is the following characterization of the absolutely continuous finite measures:

(2) $\lambda \in M(G)$ is absolutely continuous iff its left translates are equiregular, i.e., for any compact $K \subset G$ and $\varepsilon>0$ there is an open $W \supset K$ with $|\lambda|(x W \mid x K)=|\lambda|(x(W \backslash K))<\varepsilon$ for all $x$ in $G$. (In fact we only need this for $|\lambda|$-null $K$.)

In proving (1) we may as well take $\mu \geqq 0$. It suffices to see $\mu$ is absolutely continuous on the cosets of some open $\sigma$-compact subgroup, hence on any open $\sigma$-compact subgroup $H$ containing $\left\{x_{n}\right\}$. So we may as well take $G \sigma$-compact. Clearly we may add the identity as an element of $\left\{x_{n}\right\}$, and thus take $x_{1}=1$.

The main complication in our proof is the following reduction to finite measures; for a finite measure $\mu$ the proof is almost trivial. We can find an increasing sequence of compacta $K_{n}$ with union $G$ and $K_{n}^{2} \subset K_{n+1}$, and if we set $c_{n}=1+\mu\left(K_{n}\right)$ and $f=\sum_{1}^{\infty}\left(1 / n^{2} c_{n+1}\right) \chi_{K_{n}}$ then $f \mu_{x}$ is a finite measure

\footnotetext{
Received by the editors October 11, 1972.

AMS (MOS) subject classifications (1970) Primary 43A05, 28A70.

Key words and phrases. Absolute continuity, regularity of measures.

1 Work supported in part by the National Science Foundation.

${ }^{2}$ That is, regular Borel, finite on compacta.
} 
for each $x \in G$ (where $\left.\mu_{x}(E)=\mu(x E)\right)$ : for $x \in K_{m}$, some $m$, whence $x K_{j} \subset K_{j+1}$ for $j \geqq m$ and

$$
\sum_{m}^{\infty} \frac{1}{n^{2} c_{n+1}} \mu_{x}\left(K_{n}\right) \leqq \sum_{m}^{\infty} \frac{1}{n^{2} c_{n+1}} \mu\left(K_{n+1}\right) \leqq \sum_{m}^{\infty} \frac{1}{n^{2}} ;
$$

thus $\left\|f \mu_{x}\right\|=\sum_{1}^{\infty}\left(1 / n^{2} c_{n+1}\right) \mu_{x}\left(K_{n}\right)$ is finite.

Let $\nu=\sum_{1}^{\infty} 2^{-n}\left\|f \mu_{x_{n}}\right\|^{-1} f \mu_{x_{n}}$, a nonnegative finite measure. The set of compact null sets $E$ of $\nu$ is (left) translation invariant, and $\mu \ll \nu$ : for $\nu E=0$ implies $\mu_{x_{n}}(E)=0$ for all $n$ (in particular for $n=1$, and $\mu E=0$ ), so by hypothesis $\mu_{x}(E)=0$ for all $x$ in $G$, whence $\mu_{x_{n}}(y E)=0$ for all $n$ and $y$, and thus $v(y E)=0$.

But invariance of this collection of null sets implies $v \ll \sigma * v$ for any probability measure $\sigma$ on $G$, since

$$
0=\sigma * v(E)=\int v\left(x^{-1} E\right) \sigma(d x)
$$

implies $v\left(x^{-1} E\right)=0$ for some $x$, hence all $x$, and in particular $v(E)=0$; taking $\sigma$ absolutely continuous we have $\sigma * v$ absolutely continuous, so that $\nu$ and $\mu$ are also.

As is evident, the proof yields

(3) If $\mu$ is a Radon measure on $G$ and $\left\{x_{n}\right\}$ is a sequence for which $E$ compact and $|\mu|\left(x_{n} E\right)=0$ for all $n$ imply $|\mu|(x E)=0$ for all $x \in G$, then $\mu$ is absolutely continuous.

Half of (2) is of course trivial: if $\lambda$ is finite and absolutely continuous then for $\varepsilon>0$ we have a $\delta>0$ for which $m E<\delta$ implies $|\lambda|(E)<\varepsilon$, where $m$ is left invariant Haar measure. Thus given $K$ compact we can choose an open $W \supset K$ with $m(W \backslash K)<\delta$, so that $m(x(W \backslash K))<\delta$, and so obtain $|\lambda|(x(W \backslash K))<\varepsilon$ for all $x$. (This half fails for an infinite $\lambda$, e.g., for $e^{x} d x$ on $R$.)

Now suppose the translates of $\lambda$ are equi-regular. In showing $\lambda$ is absolutely continuous we can again assume $\lambda \geqq 0$ and $G \sigma$-compact. If $\lambda$ had a nonzero singular component, carried by a Baire set $E$ of Haar measure zero, then we could find [3, G, p. 287] a compact invariant subgroup $H$ of $G$ for which $G / H$ is metrizable while $E$ is a union of cosets of $H$, so the image of $E$ has Haar measure zero and thus the image $v$ of $\lambda$ has a nonzero singular component. Moreover since $H$ is compact, for any compact $K$ in $G$ and open $W \supset K H$ we can find an open $U \supset K$ with $W \supset U H \supset K H$, so that the translates of $\nu$ on $G / H$ are clearly equi-regular in view of the form of the general compact and general open set in $G / H$. Thus it will suffice to prove our assertion for $G$ metrizable and $\sigma$-compact, hence with a countable base of open sets. 
So let $\left\{W_{n}\right\}$ be a countable base of open sets closed under finite unions. We can clearly choose a sequence $\left\{x_{k}\right\}$ in $G$ for which $x_{1}=1$ and, for every $n,\left\{\lambda\left(x_{k} W_{n}\right): k \geqq 1\right\}$ is dense in $\left\{\lambda\left(x W_{n}\right): x \in G\right\}$. Suppose $K \subset G$ is compact and $\lambda\left(x_{k} K\right)=0$ for all $k$, and $x \in G$. Then for any $\varepsilon>0$ we have an open $W \supset K$ for which $\lambda(y W \backslash y K)<\varepsilon$ for all $y$ by hypothesis, and, since $\left\{W_{n}\right\}$ is closed under the taking of finite unions, we have a $W_{n}$ with $K \subset W_{n} \subset W$ so that

(4) $\lambda\left(y W_{n} \backslash y K\right)<\varepsilon, y \in G$.

Fix $n$ and choose $k$ so that $\left|\lambda\left(x_{k} W_{n}\right)-\lambda\left(x W_{n}\right)\right|<\varepsilon$. Since $\lambda\left(x_{k} K\right)=0$, (4) implies $\lambda\left(x_{k} W_{n}\right)<\varepsilon$, so $\lambda\left(x W_{n}\right)<2 \varepsilon$, and thus $\lambda(x K)<2 \varepsilon$ since $\lambda \geqq 0$. Since $\varepsilon>0$ is arbitrary, we conclude that $\lambda\left(x_{k} K\right)=0$ for all $k$ implies $\lambda(x K)=0$, all $x$, and now (3) applies to assert $\lambda$ is absolutely continuous, completing our proof of (2).

Since we actually only had to know (4) holds for $y$ in our sequence $\left\{x_{k}\right\}$, we can improve (2) to

$\left(2^{\prime}\right) \lambda \in M(G)$ is absolutely continuous iff any sequence of its left translates is equi-regular.

Some extensions of (1) and (2) are possible. For example in (1) we need only make our hypothesis for compact $E$ lying in a fixed neighborhood $V$ of the identity; one obtains absolutely continuity of $\mu$ on a neighborhood $U$ with $U^{3} \subset V$, but since the argument applies equally well to $\mu_{x}$, this yields absolute continuity. One can also take a slightly more general setting: if $\rho: H \rightarrow G$ is a continuous homomorphism of locally compact groups and $\left\{x_{n}\right\} \subset \rho(H)$ has $\left\{|\mu|\left(x_{n} E\right): n \geqq 1\right\}$ dense in $\{|\mu|(x E): x \in \rho(H)\}$ for each compact $E \subset G$ then $\mu$ vanishes on each Baire $E$ for which $\{y \in H: \rho(y) \in x E\}$ has Haar measure zero for each $x$ in $G$. (Take $\sigma$ the image on $G$ of an absolutely continuous probability measure on $H$.) The same conclusion holds if the $\rho(H)$-translates of $\mu$ are equi-regular. One can no doubt obtain analogues for a locally compact transformation group acting on a second countable locally compact space, at least for finite measures.

One way to satisfy the hypothesis of (1) (at least when $G$ is separable) is to assume $x \rightarrow|\mu|(x E)$ is always continuous; but then one can obtain the conclusion that $\mu$ is absolutely continuous in more direct (though less elementary) ways. For example

(5) If $\mu$ is finite (and one can easily reduce to that case) and $x \rightarrow \mu(x W)$ is continuous for all open $W$ then $\mu$ is absolutely continuous.

Indeed because of our continuity hypothesis, if $V$ is a compact neighborhood of 1 in $G$ then $\left\{\mu_{x}: x \in V\right\}$ is conditionally weakly compact in $M(G)$ by a result of Grothendieck [2, Theorem 3]; thus if $x_{\delta} \rightarrow 1$ in $G$ then since $\mu$ is the only weak* cluster point of $\left\{\mu_{x_{\delta}}\right\}$, it is also the only possible weak cluster point, and $\mu_{x_{\delta}} \rightarrow \mu$ weakly. Now on the closed subspace of 
$M(G)$ consisting of measures $\mu$ satisfying our continuity hypothesis, $L_{x} \mu=\mu_{x^{-1}}$ defines a weakly continuous representation $L$ of $G$, and since weakly continuous representations are strongly continuous by a result of Mirkil $[1,2.8], x \rightarrow \mu_{x}$ is norm continuous. So $\mu$ is absolutely continuous by the well-known result of Plessner, proving (5).

(Thus for $\mu$ finite, conditional weak compactness of $\left\{\mu_{x}: x \in V\right\}$, for $V$ a neighborhood of 1 implies $\mu$ is absolutely continuous.)

The cited result of Grothendieck uses Eberlein's well-known result on weak compactness, and of course is far less elementary than our earlier argument. But such results give an alternate route to (2) as well: $:^{3}$ under the hypothesis of (2) one can directly apply Grothendieck [2, Theorem 2(4)] to assert that our partial orbit $\left\{\mu_{x}: x \in V\right\}$ is again conditionally weakly compact, and the preceding paragraph yields (2). (I am indebted to Isaac Namioka for pointing out the relevance of this result of Grothendieck and the fact that our equi-regularity is half of his Theorem 2(4) for the orbit of $\mu$.)

Another consequence of the argument is the characterization of the weakly almost periodic elements of $M(G)$ under translation:

(6) The nonzero element $\mu$ of $M(G)$ has $\left\{\mu_{x}: x \in G\right\}$ conditionally weakly compact (if and) only if $G$ is compact and $\mu$ is absolutely continuous. ${ }^{4}$

We only need to see $G$ must be compact. If not, choose a compact $K \subset G$ with the restriction of $\mu$ to $K$ of norm $\geqq \frac{3}{4}\|\mu\|$. We can find $x_{n}$ in $G$ with $x_{n} \rightarrow \infty$ and $x_{n}^{-1} K \cap x_{m}^{-1} K=\varnothing$ for $n \neq m$. With $|\mu|=g \mu$, $|g| \equiv 1$, let $f$ be a function bounded by 1 with $f\left(x_{n}^{-1} y\right)=g(y)$ for $y \in K$ (or $f(x)=$ $g\left(x_{n} x\right)$ for $\left.x \in x_{n}^{-1} K\right)$ so that

$$
\begin{aligned}
\left|\int f(y) \mu_{x_{n}}(d y)\right| & =\left|\int f\left(x_{n}^{-1} y\right) \mu(d y)\right| \geqq\left|\int_{K} f\left(x_{n}^{-1} y\right) \mu(d y)\right|-\frac{1}{4}\|\mu\| \\
& =\left|\int_{K} g(y) \mu(d y)\right|-\frac{1}{4}\|\mu\| \geqq\left(\frac{3}{4}-\frac{1}{4}\right)\|\mu\| .
\end{aligned}
$$

But since $x_{n} \rightarrow \infty$ implies $\mu_{x_{n}} \rightarrow 0$ weak*, 0 can be the only weak cluster point of $\left\{\mu_{x_{n}}\right\}$, so that $\int f(y) \mu_{x_{n}}(d y) \rightarrow 0$, our contradiction.

\section{REFERENCES}

1. K. de Leeuw and I. Glicksberg, The decomposition of certain group representations, J. Analyse Math. 15 (1965), 135-192. MR 32 \#4211.

\footnotetext{
${ }^{3}$ But not to the improvement indicated in the final remark.

${ }^{4}$ Thus the almost periodic measures and weakly almost periodic measures under translation always coincide: if $G$ is compact they are precisely the absolutely continuous finite measures; if not, the trivial subspace $\{0\}$.
} 
2. A. Grothendieck, Sur les applications linéaires faiblement compactes d'éspaces du type $C(K)$, Canad. J. Math. 5 (1953), 129-173. MR 15, 438.

3. P. Halmos, Measure theory, Van Nostrand, Princeton, N.J., 1950. MR 11, 504.

4. R. Larsen, Measures with separable orbits, Proc. Amer. Math. Soc. 19 (1968), 569-572. MR 37 \#6689.

5. K. W. Tam, On measures with separable orbit, Proc. Amer. Math. Soc. 23 (1969), 409-411. MR 40 \#648.

Department of Mathematics, University of Washington, Seattle, Washington 98195

Current address: Mathematical Institute, University of Oxford, Oxford, England 